\title{
On-orbit Performance of MODIS Solar Diffuser Stability Monitor
}

\author{
Xiaoxiong (Jack) Xiong ${ }^{1}$, Amit Angal ${ }^{2}$, Junqiang Sun ${ }^{3}$, Jason $\mathrm{Choi}^{3}$, and Eric Johnson ${ }^{4}$ \\ ${ }^{1}$ Sciences and Exploration Directorate, NASA/GSFC, Greenbelt, MD 20771 \\ ${ }^{2}$ Science and Systems Applications Inc., 10210 Greenbelt Road, Lanham, MD 20706 \\ ${ }^{3}$ Sigma Space Co., 4801 Forbes Boulevard, Lanham, MD 20706 \\ ${ }^{4}$ Raytheon Space and Airborne Systems, El Segundo, CA 90245
}

MODIS has 20 reflective solar bands (RSBs) in the visible (VIS), near-infrared (NIR), and shortwave infrared (SWIR) spectral regions. MODIS RSBs are calibrated on-orbit by a solar diffuser (SD) with its on-orbit degradation tracked by a solar diffuser stability monitor (SDSM). The SDSM is an instrument by itself, consisting of a solar integration sphere (SIS) with 9 individual filtered detectors covering wavelengths from 0.41 to 0.94 micrometers. It functions as a ratioing radiometer with alternate observations made of the direct sunlight (Sun view) and that diffusely reflected from the SD panel (SD view) during each scheduled SD/SDSM calibration event. Since launch, Terra and Aqua MODIS and their SDSM have successfully operated for more than 12 and 10 years, respectively. The VIIRS, on-board the newly launched Suomi National Polarorbiting Partnership (NPP) spacecraft, has been designed and built with strong MODIS heritage and carries a similar and improved SD and SDSM calibration system. This paper provides an overview of SDSM design functions, its on-orbit calibration strategies and performance for both Terra and Aqua MODIS missions. Discussions of its on-orbit performance are focused on changes in SDSM detector responses over time and their impact on tracking SD on-orbit degradation. Also presented in this paper are lessons learned from MODIS SDSM and improvements made for the VIIRS SDSM, and preliminary comparisons of their on-orbit performance. 